\title{
CONVEXLY GENERATED $k$-DIMENSIONAL MEASURES
}

\author{
EDWARD F. MOORE
}

1. Introduction. Let $k$ and $n$ be integers such that $0<k \leqq n$, and let $C_{n}^{k}$ and $H_{n}^{k}$ be respectively the $k$-dimensional measures of Carathéodory and Hausdorff over Euclidean $n$-space $E_{n}$. It is already known that $C_{n}^{\mathfrak{k}}(A) \leqq H_{n}^{\mathrm{k}}(A)$ whenever $A \subseteq E_{n}$, and that equality holds whenever $k=1\left[1\right.$, p. 425], ${ }^{1}$ or $k=n$ (by a slight modification of the proof of Sard [4]). The present paper $^{2}$ gives the construction (4.4, 4.7) of a set $A^{\prime} \subseteq E_{3}$ such that

$$
0<C_{3}^{2}\left(A^{\prime}\right)<H_{3}^{2}\left(A^{\prime}\right)<\infty .
$$

It is further proved that

$$
C_{3}^{2}(A) \leqq H_{3}^{2}(A) \leqq \frac{\pi\left(1+3^{1 / 2}\right)}{2} C_{3}^{2}(A) \text { whenever } A \subseteq E_{3}
$$

This result suggests the definition of an equivalence relation (2.1) between measures. This relation had been previously mentioned, without further comment, by Hausdorff [3, p. 158]. It is shown (5.6) that this equivalence relation holds between $C_{n}^{k}, H_{n}^{k}$, and certain other $k$-dimensional measures which are derived by a certain prescribed limiting process (3.2) from functions defined only on certain convex subsets of $E_{n}$. This result enables certain proofs by Federer [2] concerning the structure of sets of finite $H_{n}^{k}$ measure to be extended to these other measures.

\section{Definitions and preliminaries.}

2.1. Definition. $\phi_{1}$ is congruous to $\phi_{2}$ if and only if $\phi_{1}$ and $\phi_{2}$ are measures over the same set $E$, and there exist finite positive numbers $K_{1}, K_{2}$ such that

$$
K_{1} \phi_{1}(A) \leqq \phi_{2}(A) \leqq K_{2} \phi_{1}(A) \text { whenever } A \subseteq E .
$$

\subsection{REMARK. Congruity is an equivalence relation.}

Presented to the Society, April 30,1949, under the title The two-dimensional measures of Caratheodory and Hausdorff; received by the editors November 28, 1949 and, in revised form, July $17,1950$.

${ }^{1}$ Numbers in brackets refer to the references at the end of the paper.

${ }^{2}$ Another set, constructed by G. Freilich (in publication) at about the same time, was proved by quite different methods to satisfy inequalities similar to (1). No examples were previously known showing $C_{n}^{k} \neq H_{n}^{k}$, and in fact Federer has shown $[2$, p. 148] the measures agree on $k$-rectifiable sets. 
2.3. REMARK. If $\phi_{1}$ is congruous to $\phi_{2}$, then sets of measure zero coincide under the two measures.

3. Gauges and generated measures. The measures considered in this paper can all be obtained by applying the same limiting process to various non-negative functions which are defined only on certain subsets of $E_{n}$. This general procedure for obtaining a measure is due to Carathéodory [1]. This is formalized below, since the results of this paper are obtained by constructions utilizing properties of the gauges.

3.1. Definition. Given a metric space $E, f$ is a gauge over $E$ if and only if $f$ is such a function that

$$
\text { domain } f \subseteq \underset{B}{E}[B \subseteq E] \text { and range } f \subseteq \underset{t}{E}[0 \leqq t \leqq \infty]
$$

3.2. Definition. Given a gauge $f$ over a metric space $E, F$ is generated by $f$ if and only if $F$ is the function defined on

$$
\underset{A}{E}[A \subseteq E]
$$

such that $F(A)$ is the limit as $r$ approaches zero of the infimum of sums of the form

$$
\sum_{j=1}^{\infty} f\left(B_{j}\right)
$$

where $B_{1}, B_{2}, \cdots$ are such members of domain $f$ that $A \subseteq \cup_{j=1}^{\infty} B_{j}$ and diameter $B_{j} \leqq r$ for $j=1,2,3, \cdots$.

3.3. Theorem. If $f$ is a gauge over a metric space, $E$ and $F$ is generated by $f$, then $F$ is a measure over $E$ and closed sets are $F$ measurable.

3.4. TheOREM. If $f$ is a gauge over a metric space $E$, domain $f$ is some subset of the set of Borel sets of $E$, and $F$ is generated by $f$, then every $A \subseteq E$ is contained in a Borel set of equal $F$ measure.

3.5. Definition. $h_{n}^{k}$ is the gauge defined on the set of all compact convex sets $B C E_{n}$ by the formula

$$
h_{n}^{k}(B)=b(k)[\text { diameter }(B)]^{k}
$$

where $b(k)$ is the Lebesgue measure of a sphere of diameter 1 in $E_{k}$.

3.6. Definition. $c_{n}^{k}$ is the gauge defined on the set of all compact convex sets $B C E_{n}$ by the formula

$$
c_{n}^{k}(B)=\sup _{\pi}\left(L^{k}(\pi(B))\right)
$$


where $L^{k}$ is $k$-dimensional Lebesgue measure over $k$-dimensional planes of $E_{n}$, and the supremum is taken over the set of all perpendicular projections $\pi$ of $E_{n}$ onto $k$-dimensional planes in $E_{n}$.

3.7. Definitions. Hausdorff measure: $H_{n}^{k}$ is generated by $h_{n}^{k}$. Carathéodory measure: $C_{n}^{k}$ is generated by $c_{n}^{k}$.

4. 2-dimensional measures over $E_{3}$.

4.1. Lemma. For any compact convex set $B \subset E_{3}$ and any $\epsilon>0$, there exist compact convex sets $B_{1}, \cdots, B_{r}$ such that

$$
B \subseteq \bigcup_{j=1}^{r} B_{j} \quad \text { and } \quad \sum_{j=1}^{r} h_{3}^{2}\left(B_{j}\right) \leqq \frac{\pi\left(1+3^{1 / 2}\right)}{2} c_{3}^{2}(B)+\epsilon .
$$

Proof. Let $D=$ diameter $(B)$. If $D=0$, take $r=1, B_{1}=B$; thus $h_{3}^{2}\left(B_{1}\right)=c_{3}^{2}(B)=0$, hence (2) holds. Thus we may assume $D>0$. Choose $a, b \in B$ such that $|a-b|=D$. Let $P_{1}$ be a plane perpendicular to the line joining $a$ and $b$. Let $f$ be the perpendicular projection function of $E_{3}$ onto $P_{1}$. Let $C=f(B)$.

If diameter $(C)=0$, then $B$ consists of the line segment of length $D$ joining $a$ to $b$. Then choose an integer $r>\pi \epsilon D^{2} / 4$, and choose $r$ closed subsegments $B_{j}$ of length $D / r$ covering $B$, giving

$$
\sum_{j=1}^{r} h_{3}^{2}\left(B_{j}\right)=\frac{r \pi(D / r)^{2}}{4}=\frac{\pi D^{2}}{4 r}<\epsilon
$$

hence (2) holds.

If $0<K=$ diameter $(C)$, then choose points $x, y \in C$ such that $|x-y|=K$. Let $P_{2}$ be the plane which is projected by $f$ onto the line passing through $x$ and $y$. Choose $u, v \in B$ such that $f(u)=x$, $f(v)=y$. Then let $Q$ be the set obtained by projecting the union of the triangles $(a, u, b)$ and $(a, v, b)$ perpendicularly into $P_{2}$. Then $L^{2}(Q)$ $=D K / 2$, but since the triangles are subsets of $B$, we have $c_{3}^{2}(B)$ $\geqq D K / 2$.

Case I. If $0<\left(1+3^{1 / 2}\right) K<D$, then let $r$ be the positive integer such that $(r-1) K \leqq D<r K$. Since $B$ is contained in a cylinder of crosssection $C$ and altitude $D$, choose compact convex subcylinders $B_{1}, B_{2}, \cdots, B_{r}$, each of altitude $K$, which cover $B$. Then for each $j, h_{3}^{2}\left(B_{j}\right) \leqq\left(\pi\left(2^{1 / 2} K\right)^{2}\right) / 4=\pi K^{2} / 2$. Hence

$$
\sum_{j=1}^{r} h_{3}^{2}\left(B_{j}\right) \leqq \frac{r \pi K^{2}}{2}
$$

but since $r K \leqq D+K<\left(1+1 /\left(1+3^{1 / 2}\right)\right) D=\left(\left(1+3^{1 / 2}\right) D\right) / 2$, we have 


$$
\sum_{j=1}^{r} h_{3}^{2}\left(B_{j}\right) \leqq \frac{\pi K\left(1+3^{1 / 2}\right) D}{4}<\frac{\pi\left(1+3^{1 / 2}\right)}{2} c_{3}^{2}(B) .
$$

Case II. If $\left(1+3^{1 / 2}\right) K \geqq D>0$, then let $r=1, B_{1}=B$, and $h_{3}^{2}\left(B_{1}\right)$ $=\left(\pi D^{2}\right) / 4 \leqq\left(\pi D K\left(1+3^{1 / 2}\right)\right) / 4 \leqq\left(1+3^{1 / 2}\right)(\pi / 2) c_{3}^{2}(B)$, hence $(2)$ holds.

4.2. Theorem. If $A \subseteq E_{3}$, then

$$
C_{3}^{2}(A) \leqq H_{3}^{2}(A) \leqq \frac{\pi\left(1+3^{1 / 2}\right)}{2} C_{3}^{2}(A) .
$$

Proof. The left-hand inequality is a previously known immediate consequence of the isodiametric inequality, and the right-hand inequality follows immediately from $3.2,3.5,3.6,3.7$, and 4.1 .

4.3. ThEOREM. If $0 \leqq k \leqq n, 0 \leqq i \leqq m, A \subseteq E_{n}, 0<M$, and $f$ is a function mapping $A$ into $E_{m}$ satisfying the condition $|f(x)-f(y)|$ $\leqq M|x-y|^{k / i}$ whenever $x, y \in A$, then $H_{n}^{k}(A) \geqq\left(b(k) / M^{i} b(i)\right) H_{m}^{i}$ (range f).

Proof. Given any sequence $B_{1}, B_{2}, \cdots$ as in 3.2 , then the sequence $C_{1}, C_{2}, \cdots$ such that $C_{j}$ is the closure of the convex hull of $f\left(B_{j}\right)$ satisfies range $f \subseteq \bigcup_{j=1}^{\infty} C_{j}$, diameter $C_{j}<M r^{k / i}$ for all $j$, and

$$
\begin{aligned}
h_{m}^{i}\left(C_{j}\right) \leqq b(i)\left[\operatorname{diameter}\left(C_{j}\right)\right]^{i} & \leqq b(i)\left(M\left[(\operatorname{diameter}(B))^{k / i}\right]\right)^{i} \\
& \leqq \frac{M^{i} b(i)}{b(k)} h_{n}^{k}\left(B_{j}\right),
\end{aligned}
$$

hence the conclusion follows by summation over $j$ and passage to the limit.

4.4. Definition. Let $A^{\prime}$ be the set of all those points of $E_{3}$ whose three coordinates are between 0 and 1 and have an octal expansion in which only the digits $0,1,2,3$ occur.

4.5. Theorem. $0<H_{3}^{2}\left(A^{\prime}\right)<+\infty$.

Proof. Let $G$ be the set of all numbers of the form

$$
\sum_{i=1}^{\infty} a_{i} \cdot 8^{-i}
$$

$a_{i}=0,1,2$, or 3 for $i=1,2,3,4, \ldots$ Since the digit 7 is excluded, every point of $G$ has a unique representation in the form (3).

Let $f$ be the function on $G$ onto the closed unit interval such that

$$
f\left(\sum_{i=1}^{\infty} a_{i} \cdot 8^{-i}\right)=\sum_{i=1}^{\infty} a_{i} \cdot 4^{-i}
$$


Then if $u, v \in G$ and $u>v$, let

$$
u=\sum_{i=1}^{\infty} a_{i} \cdot 8^{-i}, \quad v=\sum_{i=1}^{\infty} b_{i} \cdot 8^{-i}, \quad m=\inf \underset{i}{E}\left[a_{i}>b_{i}\right\rfloor ;
$$

then

$$
\begin{aligned}
u-v & =\sum_{i=1}^{\infty}\left(a_{i}-b_{i}\right) \cdot 8^{-i} \geqq\left(a_{m}-b_{m}\right) \cdot 8^{-m}-\sum_{i=m+1}^{\infty} 3 \cdot 8^{-i} \\
& \geqq 8^{-m}-(3 / 7) \cdot 8^{-m}=(4 / 7) \cdot 8^{-m}, \\
f(u)-f(v) & =\sum_{i=1}^{\infty}\left(a_{i}-b_{i}\right) \cdot 4^{-i} \leqq\left(a_{m}-b_{m}\right) \cdot 4^{-m}+\sum_{i=m+1}^{\infty} 3 \cdot 4^{-i} \\
& \leqq 3 \cdot 4^{-m}+4^{-m}=4 \cdot 4^{-m} .
\end{aligned}
$$

Thus

$$
|f(u)-f(v)| \leqq(14)^{2 / 3}|u-v|^{2 / 3} \quad \text { for all } u, v \in G .
$$

Let $g$ be the function on $A^{\prime}$ onto the unit cube $J$ in $E_{3}$ such that

$$
g(x)=\left(f\left(x_{1}\right), f\left(x_{2}\right), f\left(x_{3}\right)\right) \quad \text { for all } x=\left(x_{1}, x_{2}, x_{3}\right) \in A^{\prime} .
$$

Then $g$ satisfies the Lipschitz condition

$$
|g(x)-g(y)| \leqq 14^{2 / 3}|x-y|^{2 / 3} \text { for all } x, y \in A^{\prime},
$$

and since, by $[4], H_{3}^{3}(J)=1$, we conclude from 4.3 that $H_{3}^{2}\left(A^{\prime}\right)>0$.

To obtain the other inequality, for each $r>0$, choose $m$ so that $8^{-m} 3^{1 / 2}<r$ and note that $A^{\prime}$ is contained in the $64^{m}$ cubes $B_{j}$ with side length $8^{-m}$, corresponding to the $64^{m}=4^{3 m}$ admissible combinations of the first $m$ digits of the coordinates. Each of these cubes has diameter $8^{-m} 3^{1 / 2}<r$, and

$$
\sum_{i=1}^{64^{m}} h_{3}^{2}\left(B_{j}\right) \leqq \frac{\pi\left(3^{1 / 2} \cdot 8^{-m}\right)^{2}}{4} \cdot 64^{m}=3 \pi / 4,
$$

hence $H_{3}^{2}\left(A^{\prime}\right) \leqq 3 \pi / 4<\infty$.

4.6. Lemma. If $A^{*}$ is a closed subset of $A^{\prime}$, and $A$ is the convex hull of $A^{*}$, then

$$
c_{3}^{2}(A) \leqq\left(1-2 \cdot 10^{-10}\right) h_{3}^{2}(A) .
$$

Proof. Assume $A$ has positive diameter $D$. Let $P$ be any plane and let $\pi$ be the perpendicular projection function of $E_{3}$ onto $P$. Let $\pi(A)$ $=B$.

We wish to show that 


$$
L^{2}(B) \leqq \frac{\pi D^{2}}{4}\left(1-2 \cdot 10^{-10}\right) .
$$

Let $Z$ be the boundary of $B$ in $P$. Then $Z$ is connected. Since $A \cap$ inverse $\pi(x)$ is a line segment for each $x \in Z$, the set $S=A \cap$ inverse $\pi(Z)$ is a continuum. Choose $a, b \in S$ such that $|a-b|>3^{1 / 2} D / 2$. (If no such points exist, (4) is obviously satisfied.) Then choose $i$ so that $\left|a_{i}-b_{i}\right| \geqq\left|a_{j}-b_{j}\right|$ for $j=1,2,3$, where $a_{j}$ is the $j$ th coordinate of $a$. Then $|a-b| \leqq 3^{1 / 2}\left|a_{i}-b_{i}\right|$, hence

$$
\left|a_{i}-b_{i}\right|>\frac{1}{2}\left(3^{1 / 2} D \frac{1}{3^{1 / 2}}\right)=\frac{1}{2} D .
$$

For the above choice of $i$, let $m$ be the least integer such that there exist points $x, y \in S$ for which $3 \cdot 8^{-m} / 2 \leqq x_{i}-y_{i}$. Then $\left|a_{i}-b_{i}\right|$ $<3 \cdot 8^{-(m-1)} / 2$, hence $D / 24<8^{-m}$. Fix $x, y$ satisfying the above condition. If the $(m+1)$ th digit in the octal expansion of $y_{i}$ is $0,1,2$, or 3 , let $K$ be the set of all points $z$ such that the first $m$ digits of the octal expansion of $z_{i}$ agree with those of $y_{i}$ and the $(m+1)$ th digit of the octal expansion of $z_{i}$ is $4,5,6$, or 7 . If the $(m+1)$ th digit of the octal expansion of $y$ is $4,5,6$, or 7 , let $K$ be the set of all points $z$ such that the first $m$ digits of the octal expansion of $z_{i}$ agree with those of $\left(y_{i}+8^{-m}\right)$ and the $(m+1)$ th digit of $z_{i}$ is $4,5,6$, or 7 . In either case, $K$ is the strip between two parallel planes whose distance apart is $8^{-m} / 2, A^{*} \cap($ interior $K)$ is empty, and $x$ and $y$ are on opposite sides of $K$.

Now consider the plane $P_{1}$ which is parallel to boundary $K$ and bisects $K$. Since $S$ is connected we can choose a point $z \in\left(S \cap P_{1}\right)$. Then distance $\left(z, A^{*}\right) \geqq 8^{-m} / 4>D / 96$.

Since $\pi(z) \in Z$, choose a line $J$ in $P$ such that $J$ is a supporting line of $B$ at $\pi(z)$. Then let $P_{2}=$ inverse $\pi(J)$. Then $P_{2}$ is a supporting plane of $A$ at $z$. But since $A$ is the convex hull of $A^{*}$, and distance $\left(z, A^{*}\right)$ $>D / 96$, there exists a point $w \in\left(P_{2} \cap A^{*}\right)$ such that $|z-w|>D / 96$. Hence if $r$ is the midpoint of the line segment from $z$ to $w$, then $|r-z|=|r-w|>D / 192$. By the definition of $D$ we have $|r-s| \leqq D$ and $|w-s| \leqq D$ for all $s \in A$. Supposing that $X_{1}$ and $X_{2}$ measure the angles at $r$ of the triangles $(r, z, s)$ and $(r, w, s)$ respectively, we have, by the law of cosines,

$$
\begin{aligned}
& D^{2} \geqq|z-s|^{2}=|r-s|^{2}+D^{2} / 192^{2}-(2 D / 192)|r-s| \cos \left(X_{1}\right), \\
& D^{2} \geqq|w-s|^{2}=|r-s|^{2}+D^{2} / 192^{2}-(2 D / 192)|r-s| \cos \left(X_{2}\right),
\end{aligned}
$$

but since $X_{1}$ and $X_{2}$ are supplementary, we have $\cos \left(X_{2}\right)$ $=-\cos \left(X_{1}\right)$, hence 


$$
\begin{gathered}
D^{2} \geqq|r-s|^{2}+D^{2 / 36864} \\
|r-s| \leqq D\left(\frac{36863}{36864}\right)^{1 / 2}<D \frac{99,999}{100,000} .
\end{gathered}
$$

Since this is true for any $s \in A$, we conclude by projecting into the plane $P_{1}$ that $\sup _{x \in B}|q-x|<D(99,999 / 100,000)$, where $q=\pi(r)$, and since $r \in P_{2}$ we have $q \in J$. Let $Q$ be a half-disc of radius $D / 100,000$ and center $q$ bounded by $J$ and lying outside $B$. Then $\sup _{m \in Q, x \in B}|m-x|<D(99,999 / 100,000)+D / 100,000=D$. But since we already have diameter $B \leqq D$, this gives diameter $(B \cup Q) \leqq D$. By the isodiametric inequality, we have $L^{2}(B \cup Q) \leqq \pi D^{2} / 4$. Hence $L^{2}(B)+L^{2}(Q) \leqq \pi D^{2} / 4$, and since $L^{2}(Q)=\pi D^{2} \cdot 10^{-10} / 2$, we conclude $L^{2}(B) \leqq\left(\pi D^{2} / 4\right)\left(1-2 \cdot 10^{-10}\right)$.

4.7. Theorem. $0<C_{3}^{2}\left(A^{\prime}\right) \leqq\left(1-2 \cdot 10^{-10}\right) H_{3}^{2}\left(A^{\prime}\right)<\infty$.

Proof. This follows from 3.2, 3.5, 3.6, 3.7, 4.2, 4.5, and 4.6.

5. The general case. Let $k \leqq n$ be positive integers. For each bounded convex set $B \subset E_{n}$, let $M(B)$ be the supremum of $L^{n}(S)$, where $S$ is an $n$-simplex contained in closure $B$, and let $m(B)$ be the infimum of $L^{n}(I)$, where $I$ is a closed rectangular parallelepiped (whose faces are not necessarily parallel to the coordinate planes) containing $B$. The fact that these extremal values are attained simplifies the proofs which follow.

5.1. Lemma. If $B$ is a bounded convex subset of $E_{n}$, then

$$
m(B) \leqq n ! 2^{n-1} M(B) .
$$

Proof. By induction on $n$. If $n=1$, then $m(B)=M(B)=L^{n}(B)$. Given that the conclusion is true for $E_{n-1}$, consider any bounded convex set $B \subset E_{n}$. Let $S^{\prime}$ be an $(n-1)$-simplex contained in closure $B$ which is such that $L^{n-1}\left(S^{\prime}\right)$ is as large as possible. Let $P$ be the $(n-1)$-plane which contains $S^{\prime}$. Let $\pi$ be the perpendicular projection of $E_{n}$ onto $P$. Let $B^{\prime}=\pi($ closure $B)$. By identifying $P$ with $E_{n-1}$, we can apply the inductive hypothesis to $B^{\prime}$, obtaining a rectangular parallelepiped $I^{\prime}$ and an $(n-1)$-simplex $S^{\prime \prime}$ such that $S^{\prime \prime} \subseteq B^{\prime} \subseteq I^{\prime} \subseteq P$ and $L^{n-1}\left(I^{\prime}\right) \leqq(n-1) ! 2^{n-2} L^{n-1}\left(S^{\prime \prime}\right)$.

Let $S^{*}$ be an $(n-1)$-simplex spanned by some set $X$ such that $X \subset$ closure $B$, cardinal $(X) \leqq n$, and $\pi(X)$ is the set of vertices of $S^{\prime \prime}$. Then $L^{n-1}\left(S^{\prime}\right) \geqq L^{n-1}\left(S^{*}\right) \geqq L^{n-1}\left(S^{\prime \prime}\right)$, hence $S^{\prime} \subseteq$ closure $B$ and $S^{\prime}$ satisfies $L^{n-1}\left(I^{\prime}\right) \leqq(n-1) ! 2^{n-2} L^{n-1}\left(S^{\prime}\right)$.

Let $q=\sup _{x \in B}($ distance $(x, P))$. Choose $w \in$ closure $B$ such that distance $(w, P)=q$. Let $S$ be the $n$-simplex spanned by $S^{\prime} \cup\{w\}$, 
giving $S \subseteq$ closure $B$ and $L^{n}(S)=(q / n) L^{n-1}\left(S^{\prime}\right)$, hence $L^{n-1}\left(I^{\prime}\right)$ $\leqq n ! 2^{n-2} L^{n}(S) / q$.

Let $I=\left(E_{x}[\right.$ distance $(x, P) \leqq q]$ inverse $\left.\pi\left(I^{\prime}\right)\right)$. Then $I \supseteq B, I$ is a closed rectangular parallelepiped, and $L^{n}(I)=2 q L^{n-1}\left(I^{\prime}\right)$, hence $L^{n}(I) \leqq n ! 2^{n-1} L^{n}(S)$. The conclusion then follows from the definitions of $m(B)$ and $M(B)$.

\subsection{Lemma. Suppose}

1. $k \leqq n$ are positive integers,

2. $f$ is a gauge whose domain is some subset of the set of all bounded convex subsets of $E_{n}$, and there exists a finite positive number $K$ such that $f(B) \geqq K L^{k}(S)$ whenever $S$ is a $k$-simplex of $E_{n}, S \subset$ closure $B$, and $B \in \operatorname{domain} f$,

3. $g$ is such a gauge that there exists a finite positive number $N$ such that given any rectangular parallelepiped $I \subset E_{n}$ such that $s$ is the length of the longest side of $I$, then there exists a set $C \supseteq I$ such that diameter $C \leqq N \cdot s$ and $g(B) \leqq N \cdot s^{k}$,

4. $B \in$ domain $f, \epsilon>0$.

Then there exist $B_{1}, \cdots, B_{r}$ such that

$$
B \subseteq \bigcup_{j=1}^{r} B_{j}, \quad \sum_{j=1}^{r} g\left(B_{j}\right) \leqq \frac{2 N}{k ! K} f(B)+\epsilon,
$$

and diameter $B_{j} \leqq N n^{1 / 2} \cdot \operatorname{diameter} B$ for $j=1, \cdots, r$.

Proof. Choose a rectangular parallelepiped $I$ such that $I \supseteq$ closure $B$ and $L^{n}(I)=m$ (closure $B$ ). For $i=1, \cdots, n$ let $a_{i}$ be the length of the edges of $I$ which extend in the $i$ th direction. Choose the order of enumerating these directions in such a way that $a_{1} \geqq a_{2} \geqq \cdots \geqq a_{k-1}$ $\geqq a_{k} \geqq \cdots \geqq a_{n} \geqq 0$.

Case I. If $a_{k}=0$, then $a_{j}=0$ for $j \geqq k$. Hence $I$ is a degenerate parallelepiped of dimension less than or equal to $k-1$. Choose an integer $s>a_{1}^{k} / N \epsilon$. Then subdivide $I$ into $r=s^{k-1}$ rectangular parallelepipeds $I_{j}$ such that each of their edges have length $1 / s$ times the length of the corresponding edge of $I$, hence for each $j$, there exists a set $B_{j} \supseteq I_{j}$ such that $g\left(B_{j}\right) \leqq N \cdot\left(a_{1} / s\right)^{k}$, hence

$$
\sum_{j=1}^{s k-1} g\left(B_{j}\right) \leqq \frac{N}{s} a_{1}^{k}<\epsilon .
$$

Case II. If $a_{k}>0$, then $a_{i} \geqq a_{k}>0$ for $1 \leqq i \leqq k$. Let $\pi$ be the perpendicular projection function which maps $E_{n}$ onto a $k$-plane $P$ which contains a face $I^{\prime}$ of $I$ such that the edges of $I^{\prime}$ have lengths $a_{1}, \cdots, a_{k}$. Let $B^{\prime}=\pi($ closure $B)$. Then $I^{\prime}$ is a rectangular parallele- 
piped of $P$ containing $B^{\prime}$ such that $L^{k}\left(I^{\prime}\right)$ is minimal, for otherwise this would contradict the way in which $I$ was chosen. Then applying 5.1 we obtain a $k$-simplex $S^{\prime} \subseteq B^{\prime}$ such that $L^{k}\left(S^{\prime}\right)$ $\geqq L^{k}\left(I^{\prime}\right) /\left(k ! 2^{k-1}\right)$.

Choose a set $X \subset$ closure $B$ such that cardinal $(X) \leqq n$ and $\pi(X)$ is the set of vertices of $S^{\prime}$. Then the $k$-simplex $S$ spanned by $X$ has the property that

$$
L^{k}(S) \geqq L^{k}\left(S^{\prime}\right) \geqq L^{k}\left(I^{\prime}\right) /\left(k ! 2^{k-1}\right) \geqq a_{1} \cdot a_{2} \cdots \cdot a_{k} /\left(k ! 2^{k-1}\right) .
$$

Then since $S \subseteq$ closure $B$, we have $f(B) \geqq K \cdot a_{1} \cdots \cdots a_{k} /\left(k ! 2^{k-1}\right)$.

For $i=1, \cdots, k$, let $r_{i}$ be the positive integer such that $\left(r_{i}-1\right) a_{k}$ $<a_{i} \leqq r_{i} a_{k}$. For each such $i, r_{i} a_{k}<2 a_{i}$. Subdivide the parallelepiped $I^{\prime}$ into $r=r_{1} \cdot r_{2} \cdots r_{k}$ rectangular parallelepipeds $I_{1}, \cdots, I_{r}$ such that the $i$ th edge of each parallelepiped $I_{j}$ has length $a_{i} / r_{i} \leqq a_{k}$. For each $j$, let $A_{j}=I \cap\left(\right.$ inverse $\pi\left(I_{j}\right)$ ). Then for each $j, A_{j}$ is a rectangular parallelepiped whose longest edge has length $a_{k}$. Hence there exist sets $B_{j} \supseteq A_{j}$ such that $g\left(B_{j}\right) \leqq N a_{k}^{k}$. Then

$$
\begin{aligned}
\sum_{j=1}^{r} g\left(B_{i}\right) & \leqq r \cdot N a_{k}^{k} \leqq N \prod_{i=1}^{k} r_{i} a_{k} \leqq N \cdot 2^{k} \prod_{i=1}^{k} a_{i} \\
& \leqq \frac{N 2^{k} f(B)}{K\left(k ! 2^{k-1}\right)}=\frac{2 N}{k ! K} f(B) .
\end{aligned}
$$

5.3. Leмma. If 1,2 , and 3 of 5.2 hold, $F$ is generated by $f$, and $G$ is generated by $g$, then

$$
F(A) \geqq \frac{k ! K}{2 N} G(A) \quad \text { whenever } A \subseteq E_{n} .
$$

Proof. This follows from 3.2 and 5.2.

5.4. Definition. $F$ is a convexly generated $k$-dimensional measure over $E_{n}$ if and only if there exists a gauge $f$ such that

(1) $F$ is generated by $f$;

(2) Domain $f$ is some subset of the set of all convex Borel subsets of $E_{n}$;

(3) There exists a finite positive number $K$ such that $f(B) \geqq K L^{k}(S)$ whenever $S$ is a $k$-simplex of $E_{n}, S \subseteq$ closure $B$, and $B \in$ domain $f$;

(4) There exists a finite positive number $N$ such that whenever $I$ is a rectangular parallelepiped in $E_{n}$ such that $s$ is the length of the longest edge of $I$, then there exists a set $C \supseteq I$ such that diameter $C \leqq N s$ and $f(C) \leqq N s^{k}$.

5.5. REMARK. $H_{n}^{\boldsymbol{k}}$ and $C_{n}^{\boldsymbol{k}}$ are convexly generated $k$-dimensional measures over $E_{n}$. Also sphere measure $S_{n}^{k}$ and Gillespie measure $G_{n}^{k}$ 
as defined by Federer [2, p. 122], together with several of the measures derived from other gauges suggested by Hausdorff [3], are convexly generated $k$-dimensional measures.

5.6. TheоRем. If $\phi_{1}$ and $\phi_{2}$ are convexly generated $k$-dimensional measures over $E_{n}$, then $\phi_{1}$ is congruous to $\phi_{2}$, and $A$ is $\phi_{1}$ measurable if and only if $A$ is $\phi_{2}$ measurable.

Proof. Apply 5.3 twice to obtain the congruity. The equivalence of measurability follows from 2.3, 3.3, and 3.4.

\section{REFERENCES}

1. C. Carath€odory, Über das lineare Mass von Punktmengen-eine Verallgemeinerung des Langenbegriffs, Nachr. Ges. Wiss. Göttingen (1914) pp. 404-426.

2. H. Federer, The $(\phi, k)$ rectifiable subsets of $n$ space, Trans. Amer. Math. Soc. vol. 62 (1947) pp. 114-192.

3. F. Hausdorff, Dimension und dusseres Mass, Math. Ann. vol. 79 (1918) pp. 157179.

4. A. Sard, The equivalence of $n$-measure and Lebesgue measure in $E_{n}$, Bull. Amer. Math. Soc. vol. 49 (1943) pp. 758-759.

UNIVERSITY OF ILLINOIS 